\title{
Clinical characteristics of patients with posterosuperior labral tear: a comparison with patients with other shoulder disorders
}

This article was published in the following Dove Press journal: Journal of Pain Research

\author{
Donghwi Park ${ }^{1,2}$ \\ 'Department of Rehabilitation \\ Medicine, Daegu Fatima Hospital, \\ Daegu, Republic of Korea; \\ ${ }^{2}$ Department of Phamacology, School \\ of Medicine, Kyungpook National \\ University, Daegu, Republic of Korea
}

Background: Among the various causes of shoulder pain, the glenoid labral tear is likely to be overlooked due to its ambiguous symptoms, especially if clinicians do not have a prior suspicion, making it difficult to diagnose, unlike adhesive capsulitis or a rotator cuff tear. Therefore, the aim of this study was to evaluate the clinical features of posterosuperior (PS) labral tear.

Methods: Of the patients who visited the clinic, the medical records of patients with shoulder pain who underwent shoulder magnetic resonance imaging or ultrasound after intra-articular injection (arthrosonography) were investigated retrospectively. Based on these criteria, a total of 120 patients with adhesive capsulitis, rotator cuff problem, or PS labral tear were included for analysis in this study. Results: In the physical examination, all patients with a PS labral tear were positive on the O'Brien test $(13 / 13,100 \%)$ and the external rotation (ER) test with the upper arm in the abduction position (abduction and ER [ABER] test) $(13 / 13,100 \%)$, but they were negative on other tests, such as the Hawkins-Kennedy $(1 / 13,7.69 \%)$ and Neer $(0 / 13,0.00 \%)$ tests. Except one patient, all patients with a PS labral tear were negative on the ER test with the upper arm in the neutral position (ER test) $(1 / 13,7.69 \%)$. In the clinical history, all patients with a PS labral tear had a characteristic episode of shoulder pain occurring in the excessive ABER posture.

Conclusion: In patients with characteristic physical findings (no definite loss of motion of the shoulder joint, negative in an ER test, but positive in an ABER test and O'Brien test), and with a clinical history of PS labral tear (a history of pain occurring with the excessive ABER posture), clinicians need to have a suspicion of PS labral tear and to identify the PS labral tear using MRI or arthrosonography

Keywords: labral tear, arthrosonography, glenoid labrum, adhesive capsulitis, posterosuperior labral tear

\section{Introduction}

Among various causes of shoulder pain, the glenoid labral tear is likely to be overlooked due to its ambiguous symptoms, especially if clinicians do not have a prior suspicion, making it difficult to diagnose, unlike adhesive capsulitis or a rotator cuff tear. ${ }^{1-3}$ Among the types of glenoid labral tear, it has been shown that the posterosuperior (PS) labral tear can occur easily in athletes using overhead throwing exercises with PS impingement (PSI) or nonathletes with tension overload in the rotator cuff and with repetitive shearing of the humeral head against the PS labrum. ${ }^{1,4,5}$ In the diagnosis of a PS labral tear, Park ${ }^{6}$ recently reported the usefulness of ultrasound (US) after intraarticular (IA) injection (arthrosonography) compared with MRI. In that study, the authors presented that arthrosonography of the shoulder is accurate and more reliable
Correspondence: Donghwi Park Department of Rehabilitation Medicine, Daegu Fatima Hospital, Ayangro 99 , Dong gu, Daegu, 4I 199, Republic of Korea

Tel +82539407821

$\mathrm{Fax}+82539547417$

Email bdome@hanmail.net 
than conventional US for diagnosing a PS labral tear. Therefore, we thought that a retrospective review of the detailed clinical history and physical examination in patients with a PS labral tear confirmed by shoulder arthrosonography and/ or magnetic resonance imaging (MRI) could be helpful for clinicians to identify PS labral tear when they first examine patients with a PS labral tear. Therefore, in this study, we retrospectively investigated the detailed clinical history and physical examination of patients diagnosed with a PS labral tear among patients with shoulder pain who had visited our hospital and compared their characteristics with other patients who had other causes of shoulder pain.

\section{Participants and methods}

\section{Participants}

Because individual patients were not identified, the Institutional Review Board of Daegu Fatima Hospital approved this study without the requirement of obtaining informed consent. The patient records and information were anonymized prior to analysis. Among the patients who visited our hospital, the medical records of those with shoulder pain (defined as a numerical rating scale of $>3$ ) and who had undergone arthrosonography and/or MRI were investigated retrospectively. Most patients underwent US or MRI for shoulder pain during first visit. The following patients were excluded: those who had any history of neck or shoulder surgery, cervical radiculopathy, brachial plexopathy, previous shoulder surgery, previous fracture history around the shoulder joint (humerus or clavicle fracture, and so on) or dislocation, complex regional pain syndrome, and hemiplegic shoulder pain syndrome. Based on these criteria, a total of $120(120 / 436 ; 27.5 \%)$ patients were included for analysis in this study (Figure 1).

Among the patients with shoulder pain, those with a PS labral tear were defined as patients with local pain and/or tenderness around the PS labrum (PS aspect of glenohumeral joint) and patients with a PS labral tear revealed through MRI and/or arthrosonography. ${ }^{5,6}$ Part of these patients was reported by us previously. ${ }^{6}$ Patients with adhesive capsulitis were defined as patients with a normal radiograph finding of the affected shoulder and passive loss of motion (LOM) of the glenohumeral joint $>25 \%$ or $30^{\circ}$ in at least two directions (flexion, external rotation [ER], and internal rotation) compared with the contralateral side or normal value., ${ }^{7,8}$

In addition, we investigated the initial physical examination and clinical characteristics of the early stage of adhesive

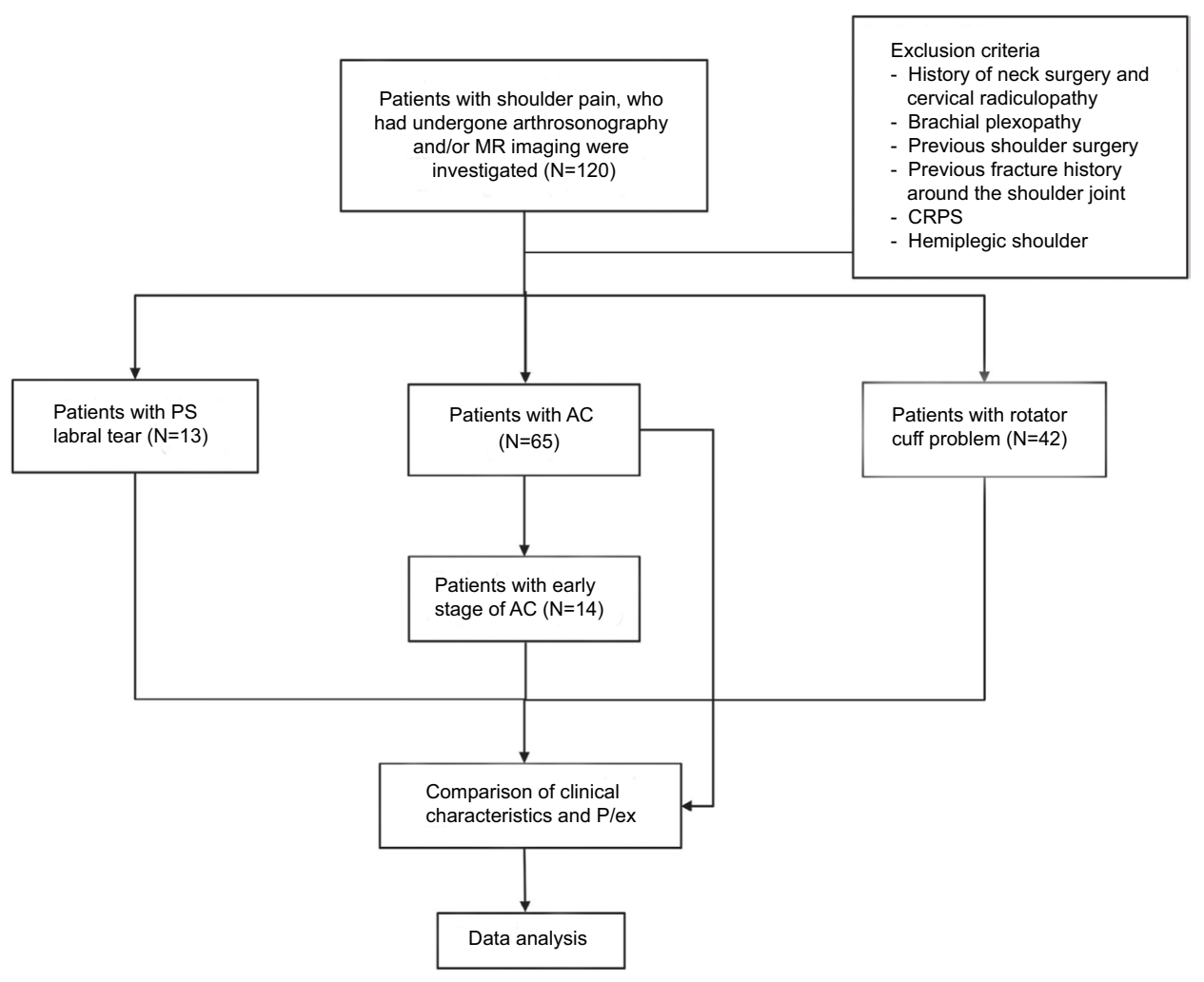

Figure I The flow chart of this study.

Abbreviations: AC, acromioclavicular; CRPS, complex regional pain syndrome; MR, magnetic resonance; P/ex, physical examinations; PS, posterosuperior. 
capsulitis, the prestage, which does not meet the definition of typical adhesive capsulitis. This is because, in the early stage, adhesive capsulitis is difficult to differentiate from a PS labral tear. The early stage of adhesive capsulitis is defined as satisfying all of the following conditions: 1) there is no typical LOM, but patients feel pain at the end range of passive motion, especially at ER, 2) patients do not show a PS labral tear on MRI and/or arthrosonography, and 3) if the patient's passive LOM worsens during follow-up and these symptoms are then satisfying the definition of typical adhesive capsulitis.

\section{Physical examination}

Before the arthrosonography and/or MRI, physical examinations were performed on the shoulder of the patient's painful side. The physical examinations were performed by a clinician with $>5$ years of experience in a musculoskeletal pain clinic. Routinely, tenderness of the acromioclavicular (AC) joint, the biceps longhead tendon and great tuberosity (insertion area of supraspinatus tendon), and the range of motion of shoulder joint were checked. Hawkins-Kennedy's test and Neer's test were performed to evaluate the shoulder impingement, and an adduction stress test was performed to evaluate the AC joint arthritis..$^{9,10}$ A positive sign in HawkinsKennedy's and adduction stress tests involves reproducing the pain of impingement. In Neer's test, reproduction of pain at the anterior edge of the acromion/lateral deltoid was considered as a positive response for impingement. In addition, an O'Brien test was performed to evaluate superior labrum anterior and posterior (SLAP) tear ${ }^{11,12}$ and ER tests with the upper arm in a neutral position (ER test) and in an abduction position (abduction and ER [ABER] test; abduction to 90-100, extension to 10-15, and maximal ER) were also performed. ${ }^{13,14} \mathrm{O}$ 'Brien test is considered as positive for a SLAP if pain felt "inside" the joint is felt to improve with the supination. However, the pain is felt "on top" in the AC joint, it is also considered positive for AC joint problem. The ER test and ABER test were performed at the patient's side with the elbow at $90^{\circ}$ of flexion. The examiner stood next to the patient on the involved side and gently externally rotated the forearm while maintaining the neutral position and the abduction position of the upper arm. ${ }^{14}$ The ER test and ABER test were considered positive if pain was produced with gentle passive ER.

And to determine whether the PSI existed, the presence of glenohumeral internal rotation deficit (GIRD) was also evaluated. ${ }^{15,16}$ A Spurling test was also performed to rule out the presence of cervical radiculopathy. ${ }^{17,18}$

\section{US evaluation}

US evaluations were performed using a standard US device (Samsung Medison, Hongchun, South Korea) and a highfrequency linear transducer (5-12 MHz). US examinations were performed by a physiatrist with $>5$ years of experience in musculoskeletal US. US evaluations were performed by the same physiatrist who performed the physical examinations. At first, the biceps tendon, AC joint, subscapularis, supraspinatus, and infraspinatus tendons were evaluated in order and the PS labrum was evaluated at the end. When examining the PS labrum, the patient sat down in front of the examiner and, then, the examiner adducted and internally rotated the arm with an elbow flexion of $90^{\circ}$. The hand on the painful side was put on the contralateral shoulder. After evaluation of the PS labrum, patients then underwent US-guided IA steroid injection (triamcinolone $40 \mathrm{mg}+$ normal saline $7 \mathrm{cc}$ $+1 \%$ lidocaine $7.5 \mathrm{cc}$, total $15 \mathrm{cc}$ ) into the glenohumeral joint. After IA injection, US evaluation of the painful shoulder was performed again to evaluate the presence of leakage through the rotator cuff (to determine whether there was a full-thickness tear of the rotator cuff tendon) and detachment of the PS labrum (to evaluate the PS labral tear). It is considered positive for PS labral tear, if detachment of the PS labrum or defined focal hypoechoic cleft was seen in arthrosonography. ${ }^{6}$ Moreover, shoulder MRI with a 3.0T Ingenia MRI system (Philips Healthcare, Best, the Netherlands) was carried out on patients with a PS labral tear who consented, to evaluate and confirm the arthrosonographic findings (Figures 2 and 3 ).

In our clinic, the US after the IA injection was performed as a routine procedure in patients with suspected adhesive capsulitis, rotator cuff problems, or a PS labral tear. This is because not only this procedure is one of the treatment methods for these patients, but also a leakage through rotator cuff in US after IA steroid injection can help to determine the full-thickness tear of the rotator cuff tear and the detachment of the PS labral tear. ${ }^{19,20}$

\section{Statistical analysis}

The results are presented in terms of numbers and percentage. To assess the reliability of US, we assessed the intra-rater and inter-rater reliabilities of the US imaging. To assess the intra-rater and inter-rater reliabilities, the stored US images of 52 patients with or without a PS labral tear were re-evaluated in regard to the presence of a PS labral tear by two clinicians who were blinded to the results of the physical examination. The intra-rater and inter-rater reliabilities of the ultrasonographic measurements of the PS labral tear were determined using intraclass correlation coefficients (ICCs) 


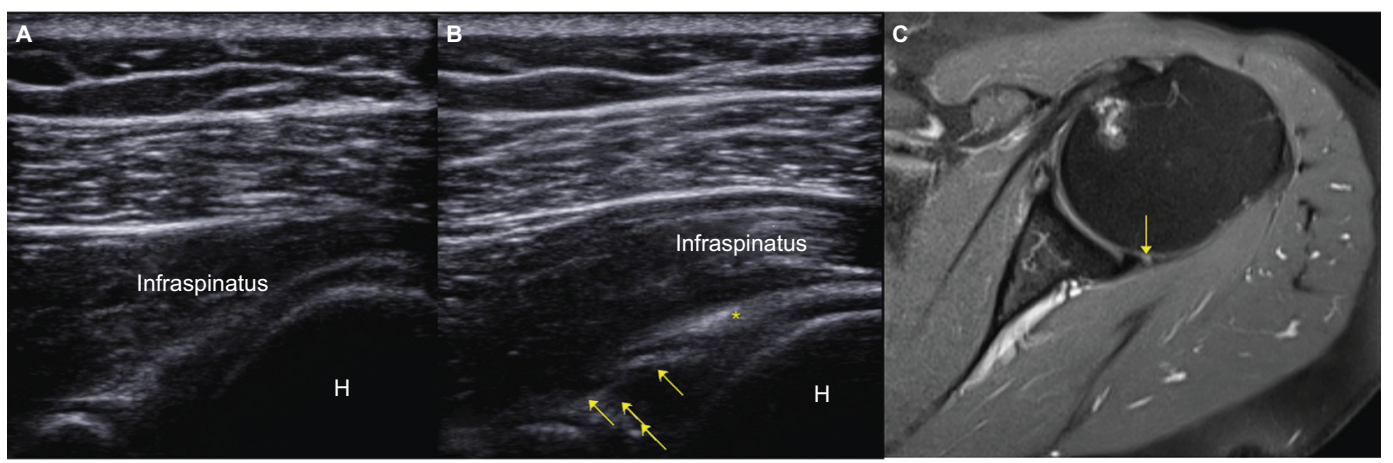

Figure 2 US and MR image of the PS labral tear.

Notes: (A) Long axis infraspinatus view (oblique axial plane view) of the PS labrum in conventional US. (B) Long axis infraspinatus view of the PS glenoid labral lesions in arthrosonography (arrows, well-defined focal hypoechoic cleft) within normal glenoid labrum (asterisk, hyperechoic homogeneous triangular area). Unlike conventional US, well-defined focal hypoechoic cleft was seen in arthrosonography. (C) Fat-suppressed T2-weight axial MR image demonstrates PS glenoid labral lesion (arrow). Abbreviations: $\mathrm{H}$, humerus; MR, magnetic resonance; PS, posterosuperior.

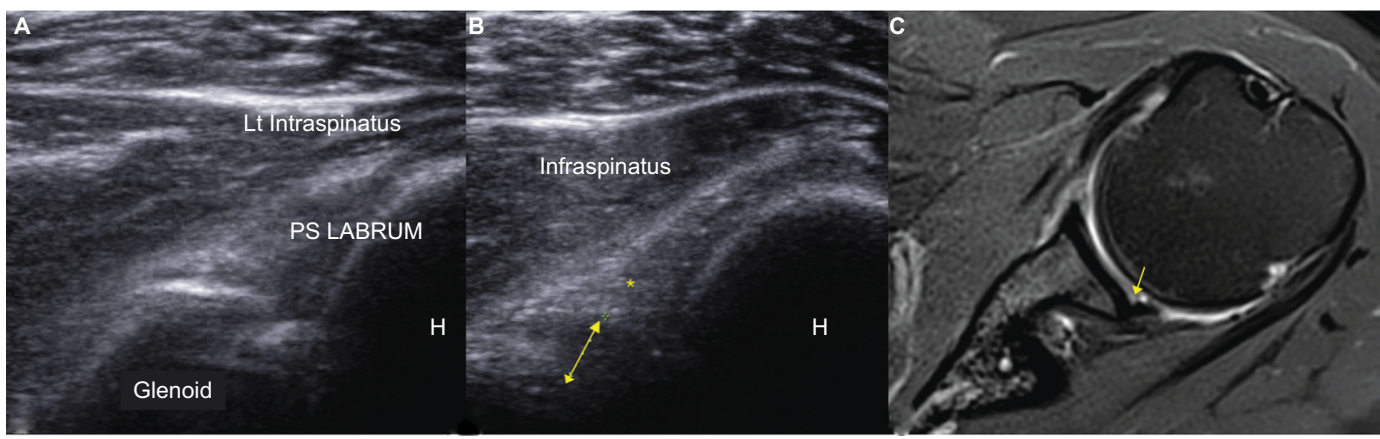

Figure 3 US and MR image of the PS labral tear.

Notes: (A) Long axis infraspinatus view (oblique axial plane view) of the PS labrum in conventional US. (B) Long axis infraspinatus view of the PS labral tear in arthrosonography (arrow, dilated hypoechoic area from the base of labrum) with normal glenoid labrum (*hyperechoic homogeneous triangular area). Unlike conventional US, well-defined focal hypoechoic cleft was seen in arthrosonography. (C) Fat suppressed T2-weight axial MR image demonstrates PS labral tear (arrow).

Abbreviations: H, humerus; Lt, left; MR, magnetic resonance; PS, posterosuperior; US, ultrasound.

Table I Demographic data of patients in this study

\begin{tabular}{lllll}
\hline & Adhesive capsulitis & Posterosuperior labral tear & Rotator cuff problem & Total \\
\hline Number (M:F) & $65(21: 44)$ & $13(1 \mathrm{I}: 2)$ & $42(23: 19)$ & $120(55: 65)$ \\
Age (years), mean \pm SD & $58.65 \pm 7.93$ & $49.92 \pm 13.26$ & $58.17 \pm 8.75$ & $56.62 \pm 9.79$ \\
Side (L:R) & $(38: 27)$ & $(2: 11)$ & $(17: 25)$ & $(57: 63)$ \\
\hline
\end{tabular}

Abbreviations: $F$, female; $L$, left; $M$, male; $R$, right.

with a corresponding $95 \%$ confidence interval (CI). To evaluate the difference of initial findings of physical examination between early stage of adhesive capsulitis and PS labral tear, the Pearson's chi-squared test was performed. All statistical analysis was performed using the SPSS software, Version 22.0 (IBM Corporation, Armonk, NY, USA).

\section{Results}

\section{Demographics}

A total of 120 patients were included in the study, with an average age of 56.62 \pm 9.79 years (range 29-78 years) (Table 1). Of whom, 65 patients had adhesive capsulitis, 13 patients had a PS labrum tear, and 42 patients had a rotator cuff problem (including impingement syndrome, rotator cuff tendon tear, and calcific tendinitis). The average age of patients with adhesive capsulitis was $58.65 \pm 7.93$ years (range 42-78 years), and their sex ratio (male:female) was 21:44. Among the patients with adhesive capsulitis, we further analyzed 14 patients with an early stage of adhesive capsulitis retrospectively. Their average age was $56.05 \pm 9.72$ years (range 42-68 years), and the sex ratio was 3:11 (Table 2). The average age of patients with a PS labral tear was $48.31 \pm 13.95$ years (range 29-66 years), and their sex ratio was 11:2. The average age of patients with a rotator 
Table 2 The differences in clinical characteristics and physical examination between patients with an early stage of adhesive capsulitis and patients with a posterosuperior labral tear

\begin{tabular}{|c|c|c|c|}
\hline Characteristics & Early stage of adhesive capsulitis & Posterosuperior labral tear & $P$-value \\
\hline Number (M:F) & $14(3: 10)$ & $13(||: 2)$ & 0.001 \\
\hline Age (years), mean $\pm S D$ & $56.05 \pm 9.72$ & $48.31 \pm 13.95$ & 0.227 \\
\hline Side (L:R) & $(3: 11)$ & $(2: 11)$ & 0.686 \\
\hline \multicolumn{4}{|l|}{ Physical examinations, n (\%) } \\
\hline O’Brien & $3(2.1)$ & $13(100)$ & $<0.001$ \\
\hline ABER test & $14(100.0)$ & $13(100)$ & 1.000 \\
\hline ER test & $14(100.0)$ & I (7.69) & $<0.001$ \\
\hline Hawkins-Kennedy's test & $3(2.14)$ & I (7.69) & 0.315 \\
\hline Neer's test & $0(0.0)$ & $0(0.0)$ & 1.000 \\
\hline Adduction stress test & $0(0.0)$ & $0(0.0)$ & 1.000 \\
\hline Tenderness on GT & $0(0.0)$ & $0(0.0)$ & 1.000 \\
\hline Tenderness on AC jt & $0(0.0)$ & $0(0)$. & 1.000 \\
\hline Tenderness on BLT & $0(0.0)$ & I (7.69) & 0.290 \\
\hline
\end{tabular}

Notes: When the tenderness was greater on one side than the other side, the tenderness was positive. ER test, ER test with the upper arm in a neutral position. Statistically significant $P$-values less than 0.05 are shown in bold.

Abbreviations: ABER; abduction and ER; AC jt, acromioclavicular joint; BLT, biceps longhead tendon; ER, external rotation; F, female; GT, great tuberosity; L, left; M, male; $\mathrm{R}$, right.

cuff problem was $58.17 \pm 8.75$ years (range $37-75$ years), and their sex ratio was 23:19 (Table 1).

\section{Reliability of US evaluation}

The ICC for intra-rater reliability was 0.982 in regard to the presence of detachment of the PS labrum or defined focal hypoechoic cleft in the arthrosonography, and the ICC (Cronbach's alpha value) for inter-rater reliability was 0.963 . The following classification scheme was used for ICC: $<0.40=$ poor, $0.40-0.59=$ fair, $0.60-0.74=$ good, and $>0.74=$ excellent. ${ }^{21}$ Thus, the measurement method for these parameters was considered to be reliable.

\section{Characteristics of patients with a PS labral tear}

Of the 120 patients, $13(10.83 \%)$ patients were diagnosed with a PS labral tear through arthrosonography and/or MRI. All 13 patients were positive on arthrosonography, and five of them were confirmed by MRI. Their sex ratio was 11:2, which was much higher in men than patients with other causes of shoulder pain. Although six patients each enjoyed golf, baseball, or tennis twice or thrice a week as recreation sports, all of them were nonathletic and none of them showed GIRD.

In the clinical history, all 13 patients with a PS labral tear had a characteristic episode of pain occurring on excessive ABER upper arm posture, such as development of posterior shoulder pain after having picked up a remote controller that was located behind them or development of posterior shoulder pain with a clicking sensation when abducting and externally rotating her shoulder by putting her arms into the sleeves to wear her tight jacket (Table 3).

\section{Early stage of adhesive capsulitis versus PS labral tear}

We investigated the clinical characteristics and initial physical examinations of patients with an early stage of adhesive capsulitis through a review of medical records. This is because it is difficult to differentiate a PS labral tear from the early stage of adhesive capsulitis by using physical examination only. To compare with a PS labral tear, an adhesive capsulitis patient who had a PS labral tear on arthrosonography (66-year-old man, flexion $165^{\circ}$, abduction $80^{\circ}$, ER at upper arm in neutral position $60^{\circ}$ ) was excluded from both the early stage of adhesive capsulitis and the PS labral tear groups. Interestingly, in both groups (the early stage of adhesive capsulitis and the PS labral tear groups), there was no definite LOM in the glenohumeral joint and most of the patients in both groups were positive on the ABER test. Moreover, in the O'Brien test, $2.14 \%(3 / 14)$ of the patients with the early stage of adhesive capsulitis were positive. However, unlike the patients with a PS labral tear, who were mostly negative in the ER test, the patients with the early stage of adhesive capsulitis tended to be positive in the ER test, although the patients of both groups were positive in the ABER test. Moreover, patients with a PS labral tear were characterized by trauma episodes of pain occurring on adopting an excessive ABER upper arm posture. In addition, Pearson's chi-squared test showed that 
Table 3 Clinical characteristics of patients with posterosuperior labral tear

\begin{tabular}{|c|c|c|c|c|c|c|}
\hline $\mathbf{P C N}$ & Sex & $\begin{array}{l}\text { Age } \\
\text { (years) }\end{array}$ & Side & Hobby & $\begin{array}{l}\text { Combined } \\
\text { pathology }\end{array}$ & Traumatic episode \\
\hline I & $\mathrm{F}$ & 58 & Right & None & & Pain occurred when putting her arms into the sleeves of her tight jacket \\
\hline 2 & $\mathrm{~F}$ & 54 & Left & None & & Pain occurred after having picked up a remote controller that was located behind her \\
\hline 3 & M & 51 & Right & Golf & & $\begin{array}{l}\text { Posterior shoulder pain occurred after having picked up a bag from the back seat of } \\
\text { the car }\end{array}$ \\
\hline 4 & M & 61 & Right & None & SST full-thickness tear & Pain occurred after the hands touched the floor when falling \\
\hline 5 & M & 63 & Right & None & $\begin{array}{l}\text { SST partial tear with } \\
\text { calcification }\end{array}$ & Pain occurred after having picked up a bag from the back seat of the car \\
\hline 6 & M & 37 & Right & None & & Pain occurred when the shoulder moved backward with heavy objects \\
\hline 7 & M & 66 & Right & Tennis & SST partial tear & Pain occurred when the arm was in the ABER posture to hit the ball during tennis \\
\hline 8 & M & 60 & Right & Tennis & SST partial tear & Pain occurred when the arm was in the ABER posture to hit the ball during tennis \\
\hline 9 & M & 32 & Right & Baseball & & Pain occurred in the ABER posture when preparing to throw the ball \\
\hline 10 & M & 29 & Right & None & & Pain occurred when putting her arms into the sleeves of her tight jacket \\
\hline $\mathrm{II}$ & M & 34 & Right & None & & Pain occurred when the shoulder moved backward with heavy objects \\
\hline 12 & M & 54 & Left & Golf & SST partial tear & Pain occurred in the follow-up motion during a golf swing \\
\hline 13 & M & 29 & Right & Golf & & Pain occurred at the posterior aspect of the shoulder joint during a high-five \\
\hline
\end{tabular}

Abbreviations: ABER, abduction and external rotation; F, female; M, male; PCN, patient case number; SST, supraspinatus tendon.

there were significant differences in O'Brien and ER tests between the two groups (Table 2).

\section{Discussion}

As mentioned earlier, among various causes of shoulder pain, the PS labral tear is likely to be overlooked due to its ambiguous symptoms, especially if clinicians do not have a prior suspicion of it, making it difficult to diagnose, unlike adhesive capsulitis or a rotator cuff tear. ${ }^{1-3}$ It is also true that a labral tear is not easy to diagnose in most local clinics where MRI is not readily available. However, in previous studies, the usefulness of arthrosonography in the diagnosis of PS labral tear was proven by comparison with MR arthrography. ${ }^{3,6}$ So, the purpose of this study was to compare the clinical characteristics of PS labral tear with other causes of shoulder pain, such as adhesive capsulitis and rotator cuff problems. This is because it can be helpful for clinicians to have a suspicion about PS labral tear when they first examine the patients with a PS labrum tear.

In fact, it is difficult to differentiate a PS labral tear from the early stage of adhesive capsulitis by using physical examination only. This is because, in both groups, there was no definite LOM in the shoulder joint and most of the patients in both groups were positive on the ABER test. Moreover, in the O'Brien test, $\sim 2.14 \%$ of the patients with the early stage of adhesive capsulitis were positive. However, unlike the patients with a PS labral tear, who were mostly negative in the ER test, the patients with the early stage of adhesive capsulitis tended to be positive in the ER test, although the patients of both groups were positive in the ABER test. Moreover, patients with a PS labral tear were characterized by trauma episodes of pain occurring at excessive ABER posture. In addition, patients with an early stage of adhesive capsulitis were more likely to feel pain in the lateral aspect of the upper arm as well as in the shoulder joint, but most of the patients with a PS labral tear tended to feel focal pain in the PS area of the glenohumeral joint. Considering these differences of between PS labral tear and the early stage of adhesive capsulitis, in the ABER and O'Brien tests, the pain in patients with a PS labral tear is more likely to develop from the labrum, which is a painsensitive tissue, but patients with an early stage of adhesive capsulitis seem to develop pain from the stretched inflamed capsule of the shoulder joint. However, it can be difficult to see a PS labral tear and adhesive capsulitis as completely different diseases, because a PS labral tear can progress to adhesive capsulitis. ${ }^{22}$ In fact, in our study, one patient had a PS labral tear revealed by arthrosonography and this actually progressed to adhesive capsulitis. Nevertheless, we believe that it is important to differentiate between adhesive capsulitis and a PS labral tear because these two pathologies have different treatment methods. Thus, we believe that our study is meaningful because it provides clinicians with clues about the initial differences in clinical characteristics between the two different pathologies, which aid accurate diagnosis.

As a part of the treatment of a PS labral tear, patients were educated about posture and exercise, as in a previous case series. ${ }^{6}$ Patients were advised to avoid excessive ABER and to start scapular stabilization exercises. In the retrospective 
review of the medical records, the patients' symptoms did not improve completely but showed significant improvement after combined IA steroid injection with scapular stabilization exercises. In adhesive capsulitis, however, IA steroid injection and capsular stretching are effective treatments unlike PS labral tear, so it is thought that deciding proper treatment by accurate diagnosis is important.

There are several limitations to this study. First, the PS labral tear was not confirmed by arthroscopy, the gold standard of diagnosis, which may result in missed mild PS labral tears in patients with shoulder pain. However, in this study, patients with a PS labral tear were diagnosed with MRI and/ or arthrosonography, which were thus proved to be useful in the diagnosis of PS labral tear in the previous studies. In addition, the mild PS labral tear may have been asymptomatic, so the lack of use of arthroscopy for diagnosis may not have had a significant impact on the results of our study which investigated the clinical characteristics of patients with a PS labral tear. Second, apart from the O'Brien test and ABER test, we could not perform more physical examinations, which are sensitive to labral tears, such as Kim's test or Jerk's test. In addition, we could not evaluate the difference of various shoulder disorders, such as SLAP tear. And, we could not evaluate the difference of psychological examination according to the cause of shoulder pain. These were due to the characteristics of this study, which is a retrospective study. Thus, a further prospective study using other physical examinations are needed in the future.

\section{Conclusion}

In patients with characteristic physical findings (patients with no definite LOM of the shoulder joint, negative in an ER test, but positive in an ABER test and O'Brien test) and with a clinical history of PS labral tear (patients with a history of pain occurring with the excessive ABER posture), clinicians need to have a suspicion of PS labral tear and to identify the PS labral tear by using MRI or arthrosonography.

\section{Acknowledgments}

This research was supported by the Basic Science Research Program through the National Research Foundation of Korea (NRF) funded by the Ministry of Science, ICT (Information and Communication Technology), and Future Planning (NRF2017R1D1A1B03033127). No commercial party having a direct financial interest in the results of the research supporting this article has or will confer a benefit upon the author or upon any organization with which the author is associated.

\section{Disclosure}

The author reports no conflicts of interest in this work.

\section{References}

1. Budoff JE, Nirschl RP, Ilahi OA, Rodin DM. Internal impingement in the etiology of rotator cuff tendinosis revisited. Arthroscopy. 2003;19(8):810-814.

2. Taljanovic MS, Carlson KL, Kuhn JE, Jacobson JA, Delaney-Sathy LO, Adler RS. Sonography of the glenoid labrum: a cadaveric study with arthroscopic correlation. AJR Am J Roentgenol. 2000;174(6): $1717-1722$.

3. Jeong WK, Ah Ryu J, Choi SH, et al. Shoulder sonography after intraarticular fluid injection for evaluation of anterior labral tears: comparison with conventional sonography. J Clin Ultrasound. 2013;41(2):94-100.

4. Burkhart SS. Internal impingement of the shoulder. Instr Course Lect. 2006;55:29-34.

5. Kirchhoff C, Imhoff AB. Posterosuperior and anterosuperior impingement of the shoulder in overhead athletes-evolving concepts. Int Orthop. 2010;34(7):1049-1058.

6. Park D. Evaluation of posterosuperior labral tear with shoulder sonography after intra-articular injection: a case series. Am J Phys Med Rehabil. 2017;96(3):e48-e51.

7. Byun SD, Park DH, Hong YH, Lee ZI. The additive effects of hyaluronidase in subacromial bursa injections administered to patients with peri-articular shoulder disorder. Ann Rehabil Med. 2012;36(1):105-111.

8. Kim DY, Lee SS, Nomkhondorj O, et al. Comparison between anterior and posterior approaches for ultrasound-guided glenohumeral steroid injection in primary adhesive capsulitis: a randomized controlled trial. J Clin Rheumatol. 2017;23(1):51-57.

9. Park KD, Kim TK, Lee J, Lee WY, Ahn JK, Park Y. Palpation versus ultrasound-guided acromioclavicular joint intra-articular corticosteroid injections: a retrospective comparative clinical study. Pain Physician. 2015;18(4):333-341.

10. Caliş M, Akgün K, Birtane M, Karacan I, Caliş H, Tüzün F, Calis M. Diagnostic values of clinical diagnostic tests in subacromial impingement syndrome. Ann Rheum Dis. 2000;59(1):44-47.

11. Owen JM, Boulter T, Walton M, Funk L, Mackenzie TA. Reinterpretation of O'Brien test in posterior labral tears of the shoulder. Int $J$ Shoulder Surg. 2015;9(1):6-8.

12. Green RA, Taylor NF, Mirkovic M, Perrott M. An evaluation of the anatomic basis of the O'Brien active compression test for superior labral anterior and posterior (SLAP) lesions. J Shoulder Elbow Surg. 2008;17(1):165-171.

13. Guanche CA, Jones DC. Clinical testing for tears of the glenoid labrum. Arthroscopy. 2003;19(5):517-523.

14. Wolf EM, Cox WK. The external rotation test in the diagnosis of adhesive capsulitis. Orthopedics. 2010;33(5).

15. Burkhart SS, Morgan CD, Kibler WB. The disabled throwing shoulder: spectrum of pathology Part I: pathoanatomy and biomechanics. Arthroscopy. 2003;19(4):404-420.

16. Kartus J, Perko M. The disabled throwing shoulder: spectrum of pathology. Part II: evaluation and treatment of SLAP lesions in throwers. Arthroscopy. 2004;20(3):336; author reply 336.

17. Anekstein Y, Blecher R, Smorgick Y, Mirovsky Y. What is the best way to apply the Spurling test for cervical radiculopathy? Clin Orthop Relat Res. 2012;470(9):2566-2572.

18. Park D. Application of ultrasound-guided C5 nerve root block using polydeoxyribonucleotide in traumatic $\mathrm{C} 5$ nerve root injury caused by fracture of the articular process of the cervical spine: A case report. Medicine. 2017;96(46):e8728.

19. Lee KW, Yang DS, Chun TJ, Bae KW, Choy WS, Park HJ. A comparison of conventional ultrasonography and arthrosonography in the assessment of cuff integrity after rotator cuff repair. Clin Orthop Surg. 2014;6(3):336-342. 
20. El-Dalati G, Castellarin G, Martone E, et al. Standard sonography and arthrosonography in the study of rotator cuff tears. Radiol Med. 2005;110(5-6):616-622.

21. Suzuki A, Daubs MD, Inoue H, et al. Prevalence and motion characteristics of degenerative cervical spondylolisthesis in the symptomatic adult. Spine. 2013;38(17):E1115-E1120.
22. Le HV, Lee SJ, Nazarian A, Rodriguez EK. Adhesive capsulitis of the shoulder: review of pathophysiology and current clinical treatments. Shoulder Elbow. 2017;9(2):75-84.

\section{Publish your work in this journal}

The Journal of Pain Research is an international, peer reviewed, open access, online journal that welcomes laboratory and clinical findings in the fields of pain research and the prevention and management of pain. Original research, reviews, symposium reports, hypothesis formation and commentaries are all considered for publication.
The manuscript management system is completely online and includes a very quick and fair peer-review system, which is all easy to use. Visit http://www.dovepress.com/testimonials.php to read real quotes from published authors. 